Review

\title{
Use of Proteins as Biomarkers and Their Role in Carcinogenesis
}

\author{
Paul Zarogoulidis ${ }^{\bowtie}$, Kosmas Tsakiridis ${ }^{2}$, Chrisanthi Karapantzou ${ }^{3}$, Sofia Lampaki1, Ioannis Kioumis ${ }^{1}$, \\ Georgia Pitsiou ${ }^{1}$, Antonis Papaiwannou ${ }^{1}$, Wolfgang Hohenforst-Schmidt ${ }^{4}$, Haidong Huang ${ }^{5}$, George \\ Kesisis ${ }^{6}$, Ilias Karapantzos 3 , Serafeim Chlapoutakis ${ }^{7}$, Ippokratis Korantzis ${ }^{6}$, Andreas Mpakas², Vasilis \\ Karavasilis ${ }^{7}$,Ioannis Mpoukovinas ${ }^{9}$, Qiang Li ${ }^{5}$, Konstantinos Zarogoulidis ${ }^{1}$ \\ 1. Pulmonary-Oncology, “G. Papanikolaou“ General Hospital, Aristotle University of Thessaloniki, Thessaloniki, Greece; \\ 2. Thoracic Surgery Department, "Saint Luke" Private Hospital, Thessaloniki, Greece; \\ 3. ORL-Oncology Unit, "'Saint Luke“" Private Hospital, Thessaloniki, Greece. \\ 4. II Medical Department, "Coburg” Regional Clinic, University of Wuerzburg, Coburg, Germany. \\ 5. Department of Respiratory Diseases, Changhai Hospital/First Affiliated Hospital of the Second Military Medical University, Shanghai, \\ People's Republic of China, China. \\ 6. Oncology Department, “Saint Luke“ Private Hospital, Thessaloniki, Greece; \\ 7. Cardiothoracic Surgery Department, University hospital of Ioannina, Greece; \\ 8. Department of Medical Oncology, Aristotle University School of Medicine, Thessaloniki, Greece; \\ 9. Oncology Department, "BioMedicine“" Private Clinic, Thessaloniki, Greece.
}

Corresponding author: Paul Zarogoulidis, Pulmonary Department-Oncology Unit, “'G. Papanikolaou“ General Hospital, Aristotle University of Thessaloniki, Thessaloniki, Greece. Tel: +306977271974, Fax: +302310992433, E-mail: pzarog@hotmail.com.

( ) Ivyspring International Publisher. This is an open-access article distributed under the terms of the Creative Commons License (http://creativecommons.org/ licenses/by-nc-nd/3.0/). Reproduction is permitted for personal, noncommercial use, provided that the article is in whole, unmodified, and properly cited.

Received: 2014.09.16; Accepted: 2014.10.15; Published: 2015.01.01

\begin{abstract}
Summary: Improved diagnostic methods and medical therapies are necessary for early detection and treatment and an improved prognosis. It is thus vital to both examine and evaluate the role of the various existing proteins as biomarkers in carcinogenesis and to assess the contribution of these proteins in anti-cancer activity, for consideration in therapeutic strategies. It is essential to both examine and evaluate the role of the various existing proteins as biomarkers in carcinogenesis and to assess the contribution of these proteins in anti-cancer activity, for consideration in therapeutic strategies. The purpose of this review is twofold. Firstly, it is to evaluate recent data about which proteins can be utilized as biomarkers in carcinogenesis. The proteins reviewed include: CPTP, IL-6, CCN, and SI00. Secondly, it is to evaluate the contribution of dietary proteins in cancer activity. Specifically, how whey protein, soy proteins and lectin, a phytochemical could be useful in cancer prevention and treatment.

Recent Findings: Whey protein, present in dairy products, is an excellent source of the sulphur amino acid cysteine, the rate limiting substrate in glutathione synthesis. Notably, this protein survives digestion and has been shown to have anti-carcinogenic properties in animal studies. Lectins are phytochemicals present in plant foods, and have active components which alters cancer initiation, promotion and progression. Lectins have been characterized as a useful tool in biochemistry, cell biology, immunology and in diagnostic and therapeutic purposes in cancer research. Soy proteins contain various compounds, including isoflavones, protease inhibitors and protein kinase inhibitors, which have been proven effective in tumor growth inhibition. They have therefore, been greatly emphasized in cancer prevention and treatment. It has been proved that soy food consumption was associated with decreased risk of death and recurrence of breast cancer. CPTP is a recently discovered protein whose main role is to transport CIP, a pro-inflammatory molecule. The discovery of CPTP may shine a light on the mechanism of inflammatory diseases, and hopefully offer a potential target for therapeutic purposes in cancer
\end{abstract}


research. Interleukin-6 is a multifunctional cytokine that affects the activity of cancer cells. It is involved in tumor growth, and elevated levels is associated with an increased risk of cancer. SIOOB is a well-established biomarker for malignant melanoma, and useful in assessing tumor load, stage and prognosis for patients with this disease. Other members of this family of proteins include SI00A4, which has been associated with several malignancies and SI00A2, which has been found to be decreased in some cancers. CCN are a group of regulatory proteins, located in the extracellular matrix (maricellular). They are involved in cellular adhesion, mitogenesis, chemotaxis, cell survival, and wound healing. CCN proteins are also able to modulate the signals of several proteins, which may also influence skeletal development and angiogenesis. Many of the functions of these proteins are thus also related to tumor growth. Furthermore, CCN interacts with estrogen in the development of cancer, and is implicated in some breast and ovarian cancers.

Key words: biomarkers, proteins, lung cancer.

\section{Introduction}

In previous experimental and epidemiological research, the role of the dietary fiber and fat in cancer development was the focus of study, while the influence of protein intake in carcinogenesis was barely understood. Research by Visek et al. (7) focused on the quantity of certain types of protein and amino acid content rather than its source. Later, an analysis in several studies on dairy products and tumor development indicated the importance of dietary milk proteins in cancer prevention.

Whey protein is a component of milk and constitutes approximately $20 \%$ of the bovine milk proteins (with casein constituting the other 80\%). Whey protein is also concentrated in the whey, the liquid bi-product of cheese manufacture, and can be isolated from these sources. The whey proteins including a-Lactalbumin, $\beta$-lactoglobulin, lactoferin, lactoperoxidase, immunoglobulins, and glycomacropetide offer a wide range chemical, physical and functional properties (8) as well as anti-cancer properties.(9) Importantly, the proteins can survive digestion, which allows it create a physiological effect both inside and outside of the digestive system. Specifically, these anti-carcinogenic properties are tied to the substrate amino acid cysteine, which with glutamate forms $\gamma$-glutamylcysteine, and subsequently with glycine forms the antioxidant glutathione (figure 1). In fact, cysteine is a limiting residue in glutathione synthesis, which can be readily supplied by whey protein, allowing for glutathione formation. Glutathione is important in protecting against oxidative stress from Reactive Oxygen Species (ROS). In addition it plays a role in cell growth, differentiation and apoptosis. These properties are thus protective against cancer formation.

Great emphasis in cancer prevention and treatment has been given to soy proteins. These proteins contain various compounds that have been have been proven to inhibit tumors in rats, and thought to pre- vent the formation of tumors in humans. isoflavones, a class of plant-based compounds that act as phytoestrogens, and various studies examine their individual action, as well as their interaction with cancer medication. Other agents such as Protease Inhibitors, specifically BBI, and Protein Kinase Inhibitors have been proven effective in tumor growth restriction or reversal. Also, Methionine Deficiency has been studied as the mechanism of tumor inhibition.

The Shanghai Breast Cancer Survival Study was a large, population-based cohort study of 5042 female breast cancer survivors in China. The conclusion of this study was that soy food consumption was significantly associated with a decreased risk of recurrence and death in female breast cancer patients.

Lectins are phytochemicals present in plant foods, and are also found in almost all organisms, including plants, invertebrates, bacteria and viruses.(5) Lectins have an active components which alters cancer initiation, promotion and progression. $(3,4)$ In fact, previous epidemiological studies proved that the consumption of a diet based on plants is associated with a reduced risk of developing certain types of cancer.(2) Furthermore, several different types of lectins exist, and have been showed to reduce the malignant potential of cancer cells. In addition, lectins have been characterized as a useful tool in biochemistry, cell biology, immunology and in diagnostic and therapeutic purposes in cancer research. (6)

CPTP, a recently discovered protein, has been shown to transport ceramide-1-phosphate (C1P), a lipid that helps regulate cell growth, survival, migration and inflammation. Specifically, C1P increases the production of pro-inflammatory eicosanoids, which are powerful signalling molecules that contribute to chronic inflammation, and thus diseases such as cancer, asthma, atherosclerosis and thrombosis. The discovery of CPTP thus shines light on the cellular mechanisms that contribute to these diseases, (15) and their potential role as biomarkers and agents for targeted therapy. 

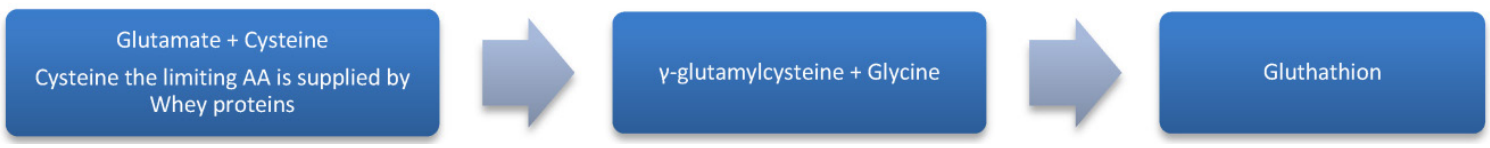

Figure I. AA; aminoacids.

Interleukin-6 (IL-6) is a glycoprotein consisting of 184 amino acids and has a molecular weight of 26 kilodaltons $(\mathrm{kDa})$. It is known as a multifunctional cytokine which, in addition to its more known roles as a proinflammatory and sclerotic agent, also affects the activity of cancer cells. Studies have indicated that it is involved in tumor growth and in distinction between benign cells and malignant cancer cells. $(16,17)$ It is shown that the levels of Interleukin- 6 are elevated in advanced cancer and that elevated levels are associated with an increased risk of cancer.(18) Because of that, IL-6 has been characterized as a prognostic marker of cancer disease. High levels of IL-6 have been linked up with increased risk of lung cancer in non-smoking women with asthma, whereas reducing IL-6 levels, by administering estrogen, has been observed to have a protective role against metastasis from hepatocellular carcinoma.

The CCN family of proteins, composed of CCN1 (CYR61), CCN2 (CTGF), CCN3 (NOV), CCN4 (WISP-1), CCN5 (WISP-2), and CCN6 (WISP-3), is a set of cysteine-rich regulatory proteins. It is believed that these matricellular proteins are involved in a wide range of functional pathways such as cellular adhesion, mitogenesis, chemotaxis, cell survival, and wound healing. CCN proteins are also able to modulate the signals of several proteins, including integrins, Wnt, Norch and bone morphogenetic protein (BMP) which may also influence skeletal development and angiogenesis.(20) Many of the functions of these proteins are thus also related to tumor growth. In fact, either overexpression or underexpression of CCN proteins has been tied to cancer development. Furthermore, estrogen seems to play an interactive role with CCN proteins in the development of cancer, especially that of breast and ovarian cancer.

S100 proteins participate in a variety of intracellular processes, including calcium homeostasis, energy metabolism, proliferation and differentiation. In addition, it is involved in inflammation, and apoptosis, as well as migration and invasion. Therefore, S100 plays a role in the healthy cell regulation, as well as cancer development. These proteins are subclassified into S100A and S100 B, and interact with a variety of other proteins, such as cytoskeletal components, enzymes, receptors, as well as transcription factors and nucleic acids.

\section{Search Methods}

We performed an electronic article search through PubMed, Google Scholar, Medscape, and Scopus databases, using combinations of the following keywords: interleukin-6, cancer, angiogenesis, CCN proteins, whey protein, lectins, S100 proteins. All types of articles (randomized controlled trials, clinical observational cohort studies, review articles, case reports) were included. Selected references from identified articles were searched for further consideration, without language limitation.

\section{Food Proteins}

\section{Whey Proteins}

Both animal and cell culture studies have proven that the anti-carcinogenic qualities of whey protein are associated with the sulphur amino acid cysteine, a substrate that combines with glutamate to form $\gamma$-glutamylcysteine. Subsequently, glycine is combined to form the antioxidant glutathione. Glutathione, is catagorized as a strong xenobiotic metabolizer, and protects against oxidative stress. Therefore, the provision of glutathione precursors by whey proteins explains the ability of dietary whey protein to protect against cancer, and reinforce humoral and cell-mediated immune responses in laboratory animals. (10)

According to the study of Fukuda et al. (21) in rats implanted with epithelioma $\mathrm{T} 8$, the consumption of milk dissipated tumor growth, by a factor of 0.4 on average. Added to this, the study in 1966 (an epidemiological study on the effect of diet, especially on milk), and the study of IARC in 1977 (dietary fiber, transit-time, steroid, colon cancer) indicated that the consumption of milk and dairy products decrease the risk of cancer. In 1983, Reddy et al. (22) indicated that yogurt reduced the number of tumor cells by a factor 0.2-0.28 in mice which were inoculated with Ehrlich ascites tumor cells. Furthermore, in the study of Nutter et al. (23) the mice which were under a milk protein formula diet, displayed an inhibition of tumor volume by a factor of 0.2-0.7. The study of Tsuru et al. (24) indicated that various types of cheese and yogurt suppressed the growth of several tumors in mice, and tumor size was reduced by a factor of $0.17-0.70$ depending on the type. According to the findings of Papenburg et al. (25) the level of tumor inhibition with the consumption of dairy products can be compared 
to the impact of a formula diet enriched with casein as a protein source. Moreover, in the study of Bounous et al. (26) it was observed that in mice which were under a casein diet, both the size and the number of dimethylhydrazine (DMH) yielded colon cancer was decreased by a factor of 0.3-0.4 compared to the Purina fed controls. Although in mice that were fed a whey protein formula diet with similar nutritional efficiency, both the number and the size of DMH induced colon tumors was reduced four fold in comparison to the Purina fed controls. DMH induced colon tumors are similar to those that have been found in humans. In 1988, Bounous et al. (27) compared the predominance of whey protein's anticancer effects to a casein diet. They showed that in terms of protein mass in bovine milk, the casein protein $(80 \%)$ contributes the predominant portion while only the remaining $20 \%$ constitutes whey protein. Furthermore, using traditional processes in order to create casein, it was found that the total amount of whey protein in the milk, which is co-precipitated along with the casein, varies from $40 \%$ to $60 \%$. They deduced that the low anti-cancer effect of casein is actually due to the small amount of whey protein that was co-precipitated with it. In other words, the level of the anticancer effect of dairy products is dependent on the protein fraction of the whey protein. The conclusion of this research is confirmed by the study by Birt et al. (28) on the longevity of Syrian hamsters. He indicated that hamsters, both female and male, which were fed a whey protein diet since the age of 4 weeks, lived longer, independent of the levels of whey protein in their diet. In the previous study of Birt et al, the survival of mice under a whey protein diet was compared to those mice that had a cysteine-enriched casein diet. After 20 weeks, the survival percentage was greater in mice with the whey protein diet than in the mice with the cysteine-enriched casein diet. The studies of Bounous et al support that the enhancement of the immune response is related to greater production of splenic glutathione in mice fed with whey protein, rather than in mice fed a cysteine-enriched casein diet.

\section{Soy Food}

According to Hawrylewicz et al.(36) factors of soy food that possibly inhibits tumor growth in rats include phytoestrogens (diadzein and genistein), deficiency of methionine, and inhibitors of protein kinase. Specifically, the soy isoflavones have been assumed to diminish breast cancer risk.(12) Due to the potential interaction between isoflavones and tamoxifen as well as to the estrogen-like effect of isoflavones, it was postulated, that consumption of soy food might be useful in breast cancer treatment.(13, 14)
The study of Troll et al. (37) postulated the following possible pathways that dietary protease inhibitors might be used to contribute to the prevention of human cancers: by blocking the formation of active oxygen species by stimulated neutrophils, inhibition of tumor promotion, and prevention of the digestion of proteins to amino acids, thus depriving rapidly growing cancer cells of essential amino acids. The pioneering studies by these investigators on the reduction of breast and skin tumors in rodents by dietary protease inhibitors stimulated extensive studies on possible beneficial anticarcinogenic effects of BBI (Bowman-Birk inhibitor) in animals and humans. In the case-control study of $\mathrm{Wu}$ et. al. (38) it was suggested that the consumption of soy-containing products may protect against breast cancer in women. Whether the protection is due to the interaction of soy isoflavones such as genistein with estrogen receptors or to other factors such as BBI and phospholipids is not known. The possible involvement of BBI is indicated by in vitro studies showing that the inhibitor suppresses the production of superoxide anion free radicals in HL-60 differentiated cells, potentiates radiation- and cisplatin-induced killing of human (breast, cervical, head and neck, lung, and ovarian) cancer cells, and also suppresses the growth of human prostate cancer xenografts in nude mice. The suppression of colon carcinogenesis in mice by BBI may be due to the observed uptake of the inhibitor by intestinal epithelial cells. Such internalization of BBI by colonic cells could facilitate the inhibition of intracellular proteases associated with the transformation of normal to malignant cells.

The protective effect of BBI in human oral cancer (leukoplakia) may be due to the inhibition of serine proteases, which cleave the neu oncogene protein, a cell surface biomarker for human cancer. A possible consequence of protease inhibition is the accumulation of the neu protein on the cell surface, leading to enhanced immune recognition of the cancer cells. This in turn permits more efficient destruction of tumors by cytotoxic lymphocytes and natural killer cells. The selective inhibition of serine proteases important for the growth of tumor cells may be a key step in cancer prevention by BBI. The effect of BBI may also be related to its ability to reach intracellular target molecules. A phase I clinical trial with 24 oral leukoplakia patients revealed that a single-dose consumption of BBI equivalent to up to 800 chymotrypsin inhibitor units was well tolerated, paving the way for further studies.

The Shanghai Breast Cancer Survival Study is a large, population-based cohort study of 5042 female breast cancer survivors in China. This study recruited women with breast cancer between 20 to 75 years old 
with a histological diagnosis made between March 2002 and April 2006. Follow up studies were completed on these women up through June 2009. Data about cancer diagnosis and treatment, lifestyle reports after cancer diagnosis and disease progression was gathered 6 months after cancer diagnosis and was reassessed at 18, 36, and 60 months. Among 5033 surgically treated breast cancer patients, during the median follow-up of 3.9 years (range, 0.5-6.2 years), 444 deaths and 534 recurrences or breast cancer-related deaths were documented. For female patients in the lowest and highest quartiles of soy protein intake, the 5-year mortality rates were $13.1 \%$ and $9.2 \%$ and 5-year recurrence rates were $13.0 \%$ and $8.9 \%$, respectively (Figure 2). For total mortality, the hazard ratio associated with the highest quartile of soy protein intake was $0.67(95 \% \mathrm{CI}=0.51-0.88)$ and for recurrence, 0.66 (95\% CI $=0.52-0.84)$, compared with the lowest quartile of intake (Table 1). Soy food intake was thus inversely connected with recurrence and mortality, meaning that patients who consumed soy foods had a decreased recurrence and mortality rate. This association was evident both in patients with estrogen receptor positive breast cancer and estrogen receptor negative breast cancer, and was present with and without tamoxifen use.

Table I. Soy protein intake and studies.

\begin{tabular}{|c|c|c|c|c|c|}
\hline \multirow[b]{2}{*}{$\begin{array}{l}\text { Soy pro- } \\
\text { tein intake }\end{array}$} & \multirow[b]{2}{*}{$\begin{array}{l}\text { No. of } \\
\text { participants }\end{array}$} & \multicolumn{2}{|c|}{ Total Mortality } & \multicolumn{2}{|c|}{ Recurrence rate } \\
\hline & & $\begin{array}{l}\text { No. of } \\
\text { events }\end{array}$ & $\begin{array}{l}\text { Hazard ratio } \\
(95 \% \text { CI })\end{array}$ & $\begin{array}{l}\text { No. of } \\
\text { events }\end{array}$ & $\begin{array}{l}\text { Hazard ratio } \\
(95 \% \mathrm{CI})\end{array}$ \\
\hline$=<5.31$ & 1254 & 117 & 1.0 & 137 & 1.0 \\
\hline $5.32-9.45$ & 1262 & 95 & $0.74(0.57-0.97)$ & 136 & $0.76(0.60-0.96)$ \\
\hline $9.46-15.31$ & 1256 & 112 & $0.68(0.52-0.89)$ & 128 & $0.67(0.52-0.86)$ \\
\hline$>15.31$ & 1260 & 120 & $0.67(0.51-0.88)$ & 133 & $0.66(0.52-0.84)$ \\
\hline
\end{tabular}

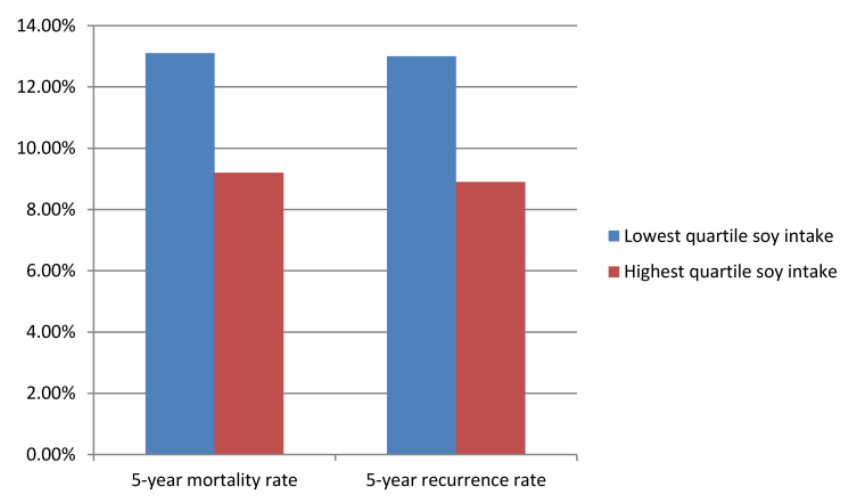

Figure 2. soy intake, mortality and recurrence rate.

\section{Lectins}

In 2000 Wang et al. (29) studied the effects of lectins on human hepatoma (H3B), human choriocar- cinoma, mouse melanoma, and rat osteosarcoma cell lines. It was indicated that pleurotus ostreatus lectin in a dose of $1.5 \mathrm{mg}$ per $\mathrm{kg}$ body for 20 days, was more efficient on sarcoma S-180 than on hepatoma H-22 tumor inhibition and it played a significant role in the survival times of the mice. He ascribed this antitumor effect to the improvement of the host immune system. According to the study of Jordinson et al.(30) Vicia Faba Agglutinin (VFA)-lectin present in broad beans reduces the malignant phenotype of colon cancer cells. The capacity of lectins to bind to carbohydrates permits glycoproteins to be expressed on cancer cells. Wheat germ agglutinin (WGA), is considered to be toxic to human pancreatic carcinoma cells in vitro. The exposure to WGA yields nuclear fragmentation, DNA release and chromatin condensation, which is consequent with apoptosis. The study of Schwarz et al. (31) suggests that the toxicity of this substance is relevant to the treatment of pancreatic cancer. Furthermore, in the study of Ganguly et al. (32) about lymphoma cell survival, it was found that WGA was capable of restricting tumor growth compared to the control group. In addition to that, in the study of Valentiner et al.(33) it was indicated that WGA influence the cell growth of human breast cancer cell lines in vitro. Another known category of lectins is MLs, which are the main compounds of mistletoe extracts. MLs display both cytotoxic effects and immunomodulatory activities. However, some recent reports have indicated that mistletoe extracts may stimulate tumor growth. The three mistletoe extracts Iscador $\mathrm{M}$ special, Iscador $\mathrm{Qu}$ and Iscador $\mathrm{P}$ were therefore assessed for anti-proliferative and stimulatory effects on a panel of 16 tumor cell lines in vitro by using a cellular proliferation assay. None of the previously mentioned was found to cause stimulation of tumor growth in the gastric, mammary, renal, uterine, or central nervous system cell lines from haematological malignancies and melanomas. Added to this, both Iscador $\mathrm{M}$ special and Iscador Qu especially showed anti-tumor activity in the mammary cancer cell line MAXF $401 \mathrm{NL}$ at a dose of $15 \mu \mathrm{g} / \mathrm{dl}$. Moreover, Maier et. al. (34) found that a little anti-tumor activity in three tumor cell lines for Iscador $M$ special, and in seven tumor cell lines for Iscador Qu special. On the contrary, Iscador P which does not contain ML-I, showed no anti-proliferative activity. In the study of Abdullaev et al.(5), a lectin which is purified by mesquite seed was studied. It was proved to have an anti-proliferative effect on cervical tumor cells (HeLa cells) (IC50 $=30 \pm 1.2 \mathrm{mg} / \mathrm{mL})$ and cell adhesion. Konno et al. (35) found that lymph node and peritoneal metastases were correlated with the strain of Gal/GalNAc-binding lectins, such as Maclura pomifera, Arachis hypogaea, and Vicia villosa, proving in 
this way that the carbohydrate expression profiles of cancer cells are relevant for their routes of dissemination and their metastatic pattern, as well as for the prognosis for colorectal cancer. According to the previously mentioned studies, lectins can be used as carriers for targeted drug delivery, depending on the glycosylation pattern of the cells and the specific lectin due to their biorecognition of specific carbohydrates.

\section{Inflammatory Proteins CPTP}

In the study of Simanshu et. al.(15), it was detected that CPTP controls levels of biologically active lipids that play a role in cell signalling. This study concluded that CPTP's main function is to transport ceramide-1-phosphate (C1P). C1P is a lipid that increases the production of pro-inflammatory eicosanoids, the powerful signalling molecules that contribute to chronic inflammation in diseases like cancer, asthma, atherosclerosis and thrombosis (figure 3). Furthermore, a decade of research from Chalfant's laboratory, supplied evidences that $\mathrm{C} 1 \mathrm{P}$ increases the eicosanoids by upregulating group IVA phospholipase $\mathrm{A} 2$, the enzyme that increases inflammation through the production of the fatty acid arachidonic acid. The release of arachidonic acid via C1P activation of CPTP was shown to increase the production of eicosanoids.

These findings help to explain the association between CPTP and thus C1P production, and the poor prognosis in breast cancer patients with elevated CPTP levels. Here it is evident that CPTP can be utilized as biomarker, and a potential target for treatment of cancer and inflammatory diseases.

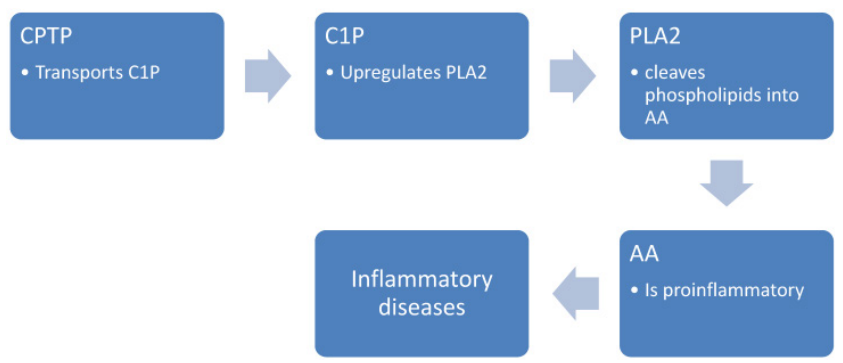

Figure 3. CIP; activate phospholipase A2, PLA2; Phospholipases A2

\section{Interleukin-6}

Ishiguro et al. (39) noticed that in lung cancer cell lines, the administration of EGFR tyrosine kinase inhibitors was a successful therapy, as the cancer cells underwent apoptosis. At the same time, IL-6 mRNA and IL-6 secretion was increased, resulting in the production of alpa-actin and collagen, and subse- quent fibrosis. It was therefore proposed that blocking IL-6 signalling could be beneficial to avoid side-effects in patients undergoing EGFR-TKI treatment. In the study by Gao et al.(19), the tyrosine-phosphorylated STAT3 signalling pathway was found to be overexpressed in cell lines that had activating mutations in the tyrosine kinase domain of EGFR. These cell lines had raised IL-6 levels and blockade of IL-6/gp130/JAK pathway, leading to an increase in the phosphorylated STAT3. Phosphorylated STAT3 is an important mediator of the effects of IL-6, having various effects, notably including cell-division. In the study of He et al.(40), increased levels of IL-2, IL-6 and tumor necrosis factor- $a$ (TNF- $\alpha$ ) have been observed in ocular metastasis, demonstrating a relation between elevated levels of IL- 6 and the metastasis.

Oh et al. (41) concluded that the biological activity of transglutaminase 2 (TG2) is correlated to that of IL-6. In breast cancer cell lines with knocked down TG2 and IL-6, experiments were carried out and it concluded that TG2 is an important factor in IL-6-mediated tumor aggressiveness, perhaps being an important mediator of metastasis.

In the study of Kim et al. (42), many agents were investigated in a brain metastasis model IL- 6 and several other proteins. It was found that among others, IL-6 levels were increased. In the study of Valles et al. (43) it has been observed that IL-6 activates Glutathione (GSH). GSH is a molecule that protects the cells against free radical damage. This helps the cancer cells to avoid undergoing apoptosis. Increased levels of the stress-related hormones corticosterone, noradrenalin and $\mathrm{ACTH}$, were shown to lead to an increased production IL-6 and thus metastasis growth.

In the study of Wang et al., estrogen administration was observed to have a protective role against metastasis from hepatocellular cancer by reducing the levels of IL-6 and hepatocellular growth factor (HGF).

\section{CCN}

A study of Xie et al.(44) observed that overexpression of Cyr61(CCN1), CTGF(CCN2), Nov(CCN3) and WISP-1(CCN4) was present in 39\%, 55\%, 11\% and $46 \%$ of primary breast tumors, respectively (figure 4 ). The presence of an Estrogen receptor (ER) was found to be a valuable predictor for Cyr61 expression, whereas HER-2/neu status was a key factor for WISP-1 expression. In this study, the stage of the disease, tumor size and lymph node status, were all significantly associated with the expression of Cyr61, CTGF and WISP-1. Another analysis in a cohort of 122 human breast tumors along with 32 normal breast tissues showed a higher level of Cyr61, but low levels of CTGF and Nov in tumors compared with normal 
tissue. In addition to that, increased levels of Cyr61 were related to a poor prognosis, lymph node involvement, metastasis and mortality. On the other hand, both CTGF and Nov levels were lower in cases with poor prognostic indications. Probably, there would be an association between estrogenic action and Cyr61 expression. In the study of Sampath et. al. (45), it was demonstrated in ER (+) MCF-7 human breast cancer cells that estradiol can induce both Cyr61 mRNA and protein. Aside from this, the levels of Cyr61 protein were found to be higher in breast tumors that were ER $(+) /$ EGF-receptor $(+)$ than those that were ER (-)/EGF-receptor(+). However, Tsai et al. (46) indicated that Cyr61 was able to induce estrogen-independence and anti-estrogen resistance, and to promote invasiveness, all of which are characteristics of an aggressive breast cancer phenotype. This result is similar to the study of Lin et al., who observed a remarkable resistance to apoptosis in MCF-7 cells that overexpressed Cyr61 against chemotherapeutic agents.

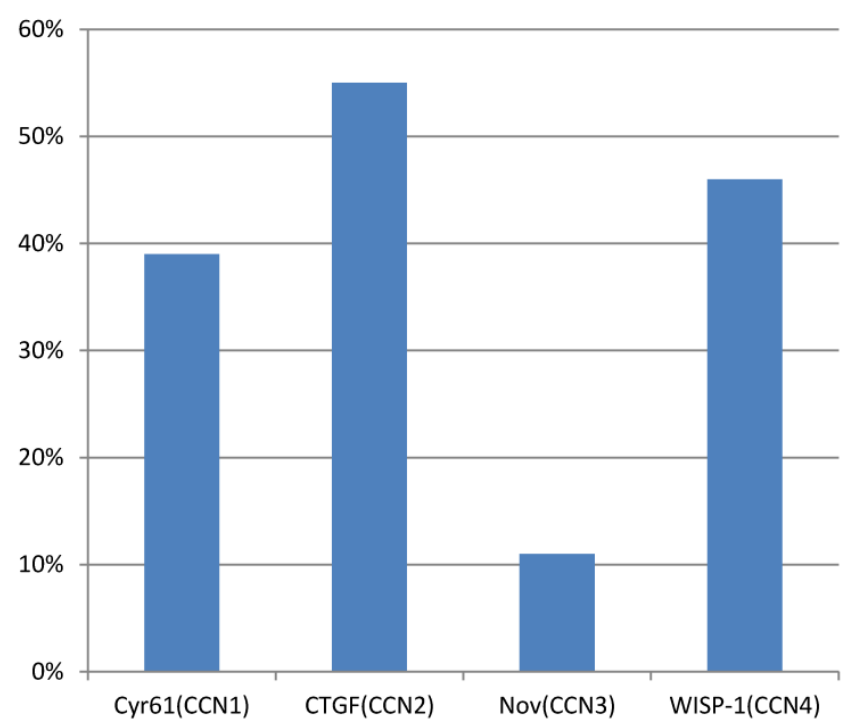

Figure 4. Overexpression in primary breast tumors. $\mathrm{CCNI}$; cysteine-rich angiogenic protein 6I, CCN2; connective tissue growth factor, CCN3; nephroblastoma overexpressed, CCN4; WNTI inducible signaling pathway protein-I.

Contrary to breast cancer, in cases of endometrial cancer things are different. Watari et al. (47) documented poor survival of patients with endometrial carcinoma of endometrioid subtype that over-expressed Cyr61. Nevertheless, Chien et al. (48), revealed a decreased level of Cyr61 in endometrial tumors compared to normal endometrium. Also, Cyr61 may play a significant role in ovarian carcinogenesis. In the study of Gery et al. (49) it was proven that estrogens increased mRNA and protein levels in ovarian cancer cells.
As far as gastric cancer is concerned, Maeta et. al. (50), found that Cyr61 expression was significantly lower in the advanced state of malignancy. Also, they noticed an inverse correlation between the expression of Cyr61 and MMP-7. Contrary to that, Lin et al. demonstrated a tumor promoting role of Cyr61 in gastric cancer cell invasion. Their results showed that Cyr61 promoted gastric cancer cells' invasive ability via hypoxia-inducible factor-1a (HIF-1a)-dependent upregulation of plasminogen activator inhibitor-1 (PAI-1). In the study of Sin et al. (51), it was mentioned that Cyr61 promoted growth in glioma cells. In addition, the study by Xie et al. (44) showed that primary gliomas exhibited a significant association between expression of both Cyr61 and CTGF vs. tumor grade.

According to Bleau et al. (52), CCN3 or Nov proteins exhibit tumor suppressive abilities. Manara et al. (53) observed an association between CCN3 expression and tumor differentiation in myosarcoma and cartilage tumors, whereas in Ewing's sarcoma, the expression seemed to be associated with a higher risk to develop metastases. On the contrary, overexpression of CCN3 was shown in metastatic melanoma cells compared with cells of the primary tumor, and xenotransplantation in immune-deficient mice showed a higher metastatic potential of melanoma cells over-expressing CCN3.

\section{Biomarkers \\ S 100}

S100B serum levels have been assessed in patients with melanoma at different stages, and have been shown to increase in a stage-dependent manner. Levels of S100B are greater in VI grade melanoma and they have been related to metastases. Added to this, Heizmann et al. (54) and Harpio et al. (55), found that there is a correlation between high serum levels of S100B and reduced survival. As S100B is able to reflect tumor load, stage and prognosis, it can be used for diagnosis and for the staging of malignant melanoma in a clinical setting.

Increased expression of S100A4 at both protein and mRNA level has been associated with many malignancies such as bladder, breast, colorectal, thyroid, astrocytic, gastric, lung and prostate cancer. Also, expression of S100A4 is inversely related to expression of E-cadherin, a molecule with an important role in cell-cell adhesion, in some cancers including melanoma and lung cancers. According to Davies et al.(56), overexpression of S100A4 proteins is associated with metastases and reduced survival. Previous studies have shown that overexpression of S100A4 can promote metastases in rodent model systems of 
breast cancer and metastases of bladder cancer in animal models. In particular LIoyd et al. (57) demonstrated that transfixion of rodent or human S100A4 into non-metastatic mammary tumor cells resulted in the acquisition of metastatic properties when re-introduced into the rodents. This supports the suggestion of a direct role for S100A4 in the promotion of metastasis.

S100A2 expression is decreased in many cancers included prostate, lung cancer, oral cancer and breast cancer. According to Suzuki et al. (58) and Kyriazanos et al. (59), loss of expression has been associated with poorer prognosis and shorter survival. In the study of Tsai et al. (60), it was mentioned that a loss of nuclear expression of S100A2 in tumor cells is significantly related to tumor recurrence and it was suggested that this could identify patients requiring closer follow-up. S100A2 expression is absent in follicular adenomas and follicular carcinomas but is up-regulated in papillary and anaplastic carcinomas.

Moog-Lutz et al. (61) isolated a cDNA encoding the S100A7 protein and showed that the gene is expressed in many breast cancer cell lines and in breast carcinomas but not in non-cancerous tissues with the exception of skin. In the study of Salama et al.(62), it has been shown that S100A7 identifies a distinct subgroup of high grade infiltrating ductal breast carcinoma. It has also been demonstrated that S100A7 is expressed in human breast carcinoma cell line MDA-MB-468 and its expression is associated with increased cell migration and invasion but reduced tumor size. This has been associated with upregulation and downregulation of matrix metallopepdidasese 13(MMP13) and vascular endothelial growth factor (VEGF), respectively, and supports the association between high expressions of S100A7 in human breast carcinomas with increased angiogenesis.

In the study of Salama et al. (62), it has been demonstrated that normal breast myoepithelial cells upregulate S100A9 in breast cancer cells and this leads to reduced tumor cell proliferation. Also in the study of Rafii et al. (63), it was indicated that primary tumors secrete soluble factors, including VEGF-A, TGFb and TNFa, which induce expression of S100A8 and S100A9 in the myeloid and endothelial cells within the lung prior to tumor metastasis.

According to Kondo et al.(64) and Memon et al. (65) S100A11 expression in both bladder and renal carcinoma is related to tumor suppression. On the contrary, according to Rehman et al.(66) and Cross et al. (67), S100A11 is regarded as being tumor promoter in both prostate and breast cancer.

\section{Conclusion}

From the abovementioned elements, we can assert that proteins play an inevitable significant role in cancer either in treatment or in deterioration of the patient. To begin with, whey proteins offer a wide range of chemical, biological and physical properties. It has been indicated that whey protein offer protection to the neonate, including anti-cancer action. In the study of Mclntosh et al.(9), it was proven that total dietary whey protein has a protective effect against the development of colon cancer in rats, when compared with other proteins such as casein, soy and meat. In addition to that, whey protein diet appeared to reduce the production of potentially carcinogenic metabolites in the gut. Preliminary results suggest that diets supplemented with lactoferrin or with b-lactoglobulin reduce the number of aberrant crypt cells. A possible mechanism for the protective influence of whey centers on the ability of these sulphur amino acid-rich proteins to protect DNA by enhancing its methylamine status. There have been made considerable efforts for the development of food products in order to provide protective protein into the diet. The findings of all the researches could also have implications for the perception and ultimately the consumption of all dairy products. (26)

Another category of proteins is lectins, which are considered to be markers for tumor cell recognition, mitogenic cytotoxicity, and apoptosis. What is more, lectins modulate the growth, proliferation, and apoptosis of premalignant and malignant cells in vitro and in vivo. In the study of Ohba et al.(68) it was indicated that tetrameric lectins probably have the greatest clinical potential, despite the fact that they show lower cytotoxicity than the dimeric lectins. Lectins have antitumor and anticarcinogenic activities that could be of benefit in cancer treatment. Gabius et al.(69), showed that lectin application has been shown to stimulate tumor growth in certain cell lines, histocultures of human tumors, and in 2 animal models using chemical carcinogenesis or tumor transplantation. Lectins inhibit DNA, RNA, and protein synthesis of malignant cells but not of normal cells. It is clear that lectins induce apoptosis. Controlled clinical trials are necessary to reach a clear conclusion about the efficiency and clinical benefit of standardized lectins.

Among breast cancer survivors, the estrogen-like effect of soy isoflavones and conflicting data from in vivo and in vitro studies regarding the role of soy constituents in stimulating cell proliferation have raised concerns about the safety of soy food consumption, while soy constituents have been shown to have anticancer properties and improve cardiovascular and bone health. (12) 
Many foods, like beverages, nutrition bars, yogurt, baked goods, meal replacements, and confections contain concentrated soy isoflavones. Exposure to isoflavones is becoming omnipresent, which increases the worry about soy food consumption among the promptly increasing population of breast cancer survivors. As we know isoflavones and tamoxifen bind to estrogen receptors and because of the contradictory results from in vivo and in vitro studies, there is concern that soy isoflavones may influence the effectiveness of used adjuvant treatment for breast cancer. In the Shanghai Breast Cancer Survival Study it was observed that soy food intake was connected with improved survival regardless of use of tamoxifen, while tamoxifen use was related to improved survival to the women who had low or moderate levels of soy food intake. Tamoxifen was not related to improvement of survival rates in the women who had the highest level of soy food intake. The women who had the highest level of soy food intake and no administration of tamoxifen had a lower risk of mortality and a lower recurrence rate than women who had the lowest level of soy food intake and used tamoxifen, proposing that high soy food intake and tamoxifen use may have a comparable affection on breast cancer outcomes. (70)

CPTP is a newly discovered protein that controls the levels of the pro-inflammatory C1P. It is hoped that the discovery of CPTP will lead to exciting new developments within the treatment of these inflammatory conditions, including cancer.

The increased plasma levels of cytokines including C-reactive protein, IL-6 and IL- 8 have been connected with high risk for lung cancer. Nevertheless; only IL-8 has been identified as an early lung cancer biomarker. Increased levels of IL-6 have been observed in advanced stage cancer in many types of cancer. In the study of Seow et al.(71), IL-6 levels have been associated with coronary heart disease. In addition, different pro-inflammatory and fibrogenic cytokines have been researched in correlation to radiation-induced lung toxicity (RILT) and it was observed that low pre-treatment levels of IL-8 were correlated with the development of RILT. Also, it has been suggested that elevated levels of circulating IL-6 is a prognostic factor for poor treatment response.

The roles of $\mathrm{CCN}$ proteins are compound in cancer. The expression levels may differ depending on the carcinogenesis of specific sites and types. CCN proteins may affect tumor progression by cell survival, cell apoptosis, angiogenesis and also by modulating different signalling pathways like Wnt, TGF-ßand IGF system. Consequently, appreciating the effects of members of CCN family is significant in order to understand their biological significance and develop- ment of therapeutic strategies. (72-76) There has been a remarkable growth in the interest of S100 proteins in cancer. It has been indicated that $\mathrm{S} 100$ proteins have a variety of intracellular and extracellular roles, and that disruption to any one of these functions may contribute to carcinogenesis. These proteins play a major role in tumor metastasis by interacting with a many different proteins, including matrix metalloproteinases, cytoskeletal proteins, p53, Jab1, Cox-2 and BRCA1. S100B is already useful in a clinical setting and there is a strong belief that other members of the S100 family will emerge as important biomarkers in the future and may provide new therapeutic opportunities. (62) Protein intake assists the defence mechanisms in an immunomodulatory aspect (77), moreover; protein intake has been found to prolong survival in several diseases (78), including cancer (79).

\section{Conflict of Interest}

The author(s) confirm that this article content has no conflicts of interest.

\section{References}

1. Edwards BK, Noone AM, Mariotto AB, et al. Annual Report to the Nation on the status of cancer, 1975-2010, featuring prevalence of comorbidity and impact on survival among persons with lung, colorectal, breast, or prostate cancer. Cancer 2014;120:1290-314.

2. Block G, Patterson B, Subar A. Fruit, vegetables, and cancer prevention: a review of the epidemiological evidence. Nutr Cancer 1992;18:1-29.

3. Boland $\mathrm{CR}$, Martin MA, Goldstein IJ. Lectin reactivities as intermediate biomarkers in premalignant colorectal epithelium. J Cell Biochem Suppl 1992;16G:103-9.

4. Temple NJ. Fruits, vegetables, and cancer prevention trials. J Natl Cancer Inst 1999;91:1164.

5. Abdullaev FI, Gonzalez de Mejia E. Inhibition of colony formation of Hela cells by naturally occurring and synthetic agents. Biofactors 1995;5:133-8.

6. Van Damme EJ, Smeets K, Engelborghs I, et al. Cloning and characterization of the lectin cDNA clones from onion, shallot and leek. Plant Mol Biol 1993;23:365-76.

7. Visek V, Mest'ak J. [Reconstruction of an amputated fingertip using an island flap]. Rozhl Chir 1986;65:813-7.

8. Smithers GW, Ballard FJ, Copeland AD, et al. New opportunities from the isolation and utilization of whey proteins. J Dairy Sci 1996;79:1454-9.

9. McIntosh GH, Regester GO, Le Leu RK, et al. Dairy proteins protect against dimethylhydrazine-induced intestinal cancers in rats. J Nutr 1995;125:809-16.

10. Parodi A, Drosera M, Barbieri L, et al. Anti-ribosomal antibodies and psychosis in patients with cutaneous lupus erythematosus. Acta Derm Venereol 1998;78:307.

11. Parodi A, Cozzani E, Chorzelski TP, et al. A molecule of about $70 \mathrm{kd}$ is the immunologic marker of chronic ulcerative stomatitis. J Am Acad Dermatol 1998;38:1005-6.

12. Friedman M, Brandon DL. Nutritional and health benefits of soy proteins. J Agric Food Chem 2001;49:1069-86.

13. Liu F, Zhuang BC, Zhang JS, et al. [Construction and analysis of soybean genetic map]. Yi Chuan Xue Bao 2000;27:1018-26.

14. Horn-Ross PL, John EM, Canchola AJ, et al. Phytoestrogen intake and endometrial cancer risk. J Natl Cancer Inst 2003;95:1158-64.

15. Simanshu DK, Kamlekar RK, Wijesinghe DS, et al. Non-vesicular trafficking by a ceramide-1-phosphate transfer protein regulates eicosanoids. Nature 2013;500:463-7.

16. Zarogoulidis $\mathrm{P}$, Yarmus $\mathrm{L}$, Darwiche $\mathrm{K}$, et al. Interleukin-6 cytokine: a multifunctional glycoprotein for cancer. Immunome Res 2013;9:16535.

17. Zarogoulidis $\mathrm{P}$, Yarmus L, Zarogoulidis K. New insights for IL-6 targeted therapy as an adjuvant treatment for non-small-cell lung cancer. Ther Deliv 2013;4:1221-3.

18. Wojcik E, Jakubowicz J, Skotnicki P, et al. IL-6 and VEGF in small cell lung cancer patients. Anticancer Res 2010;30:1773-8.

19. Gao H, Ward PA. STAT3 and suppressor of cytokine signaling 3: potential targets in lung inflammatory responses. Expert Opin Ther Targets 2007;11:869-80.

20. Dhar A, Ray A. The CCN family proteins in carcinogenesis. Exp Oncol 2010;32:2-9. 
21. Fukuda T, Hirayama Y, Mitani H, et al. Generation of metastatic variants of Eker renal carcinoma cell lines for experimental investigation of renal cancer metastasis. Jpn J Cancer Res 1998;89:1104-8.

22. Reddy JA, Westrick E, Santhapuram HK, et al. Folate receptor-specific antitumor activity of EC131, a folate-maytansinoid conjugate. Cancer Res 2007;67:6376-82.

23. Nutter RL, Gridley DS, Kettering JD, et al. Modification of a transplantable colon tumor and immune responses in mice fed different sources of protein, fat and carbohydrate. Cancer Lett 1983;18:49-62.

24. Tsuru S, Shinomiya N, Taniguchi M, et al. Inhibition of tumor growth by dairy products. J Clin Lab Immunol 1988;25:177-83.

25. Papenburg R, Bounous G, Fleiszer D, et al. Dietary milk proteins inhibit the development of dimethylhydrazine-induced malignancy. Tumor Biol 1990;11:129-36.

26. Bounous G, Gold P. The biological activity of undenatured dietary whey proteins: role of glutathione. Clin Invest Med 1991;14:296-309.

27. Bounous G, Kongshavn PA, Gold P. The immunoenhancing property of dietary whey protein concentrate. Clin Invest Med 1988;11:271-8.

28. Birt DF, Baker PY, Hruza DS. Nutritional evaluations of three dietary levels of lactalbumin throughout the lifespan of two generations of Syrian hamsters. J Nutr 1982;112:2151-60.

29. Wang Q, Yu LG, Campbell BJ, et al. Identification of intact peanut lectin in peripheral venous blood. Lancet 1998;352:1831-2.

30. Jordinson M, El-Hariry I, Calnan D, et al. Vicia faba agglutinin, the lectin present in broad beans, stimulates differentiation of undifferentiated colon cancer cells. Gut 1999;44:709-14.

31. Schwarz RE, Wojciechowicz DC, Picon AI, et al. Wheatgerm agglutinin-mediated toxicity in pancreatic cancer cells. $\mathrm{Br} \mathrm{J}$ Cancer 1999;80:1754-62.

32. Ganguly C, Das S. Plant lectins as inhibitors of tumor growth and modulators of host immune response. Chemotherapy 1994;40:272-8.

33. Valentiner U, Fabian S, Schumacher U, et al. The influence of dietary lectins on the cell proliferation of human breast cancer cell lines in vitro. Anticancer Res 2003;23:1197-206.

34. Maier G, Fiebig HH. Absence of tumor growth stimulation in a panel of 16 human tumor cell lines by mistletoe extracts in vitro. Anticancer Drugs 2002;13:373-9.

35. Konno A, Hoshino Y, Terashima S, et al. Carbohydrate expression profile of colorectal cancer cells is relevant to metastatic pattern and prognosis. Clin Exp Metastasis 2002;19:61-70

36. Hawrylewicz EJ, Zapata JJ, Blair WH. Soy and experimental cancer: animal studies. J Nutr 1995;125:698S-708S.

37. Troll W, Frenkel K, Wiesner R. Protease inhibitors: possible preventive agents of various types of cancer and their mechanisms of action. Prog Clin Biol Res 1987;239:297-315.

38. Wu AH, Ziegler RG, Nomura AM, et al. Soy intake and risk of breast cancer in Asians and Asian Americans. Am J Clin Nutr 1998;68:1437S-43S

39. Ishiguro $Y$, Ishiguro $H$, Miyamoto $H$. Epidermal growth factor receptor tyrosine kinase inhibition up-regulates interleukin-6 in cancer cells and induces subsequent development of interstitial pneumonia. Oncotarget 2013;4:550-9.

40. He Y, Fang J, Peng X, et al. Effects of Sodium Selenite on Aflatoxin B1-Induced Decrease of Ileac T cell and the mRNA Contents of IL-2, IL-6, and TNF-alpha in Broilers. Biol Trace Elem Res 2014;159:167-73.

41. Oh $\mathrm{K}, \mathrm{Ko} \mathrm{E}, \mathrm{Kim} \mathrm{HS}$, et al. Transglutaminase 2 facilitates the distant hematogenous metastasis of breast cancer by modulating interleukin- 6 in cancer cells. Breast Cancer Res 2011;13:R96.

42. Kim SH, Lee JE, Yang SH, et al. Induction of cytokines and growth factors by rapamycin in the microenvironment of brain metastases of lung cancer. Oncol Lett 2013;5:953-8.

43. Valles SL, Benlloch M, Rodriguez ML, et al. Stress hormones promote growth of B16-F10 melanoma metastases: an interleukin 6- and glutathione-dependent mechanism. J Transl Med 2013;11:72.

44. Xie D, Nakachi K, Wang H, et al. Elevated levels of connective tissue growth factor, WISP-1, and CYR61 in primary breast cancers associated with more advanced features. Cancer Res 2001;61:8917-23.

45. Sampath D, Winneker RC, Zhang Z. Cyr61, a member of the CCN family, is required for MCF-7 cell proliferation: regulation by 17beta-estradiol and overexpression in human breast cancer. Endocrinology 2001;142:2540-8.

46. Tsai MS, Bogart DF, Castaneda JM, et al. Cyr61 promotes breast tumorigenesis and cancer progression. Oncogene 2002;21:8178-85.

47. Watari $\mathrm{H}$, Xiong $\mathrm{Y}$, Hassan $\mathrm{MK}$, et al. Cyr61, a member of ccn (connective tissue growth factor/cysteine-rich 61/nephroblastoma overexpressed) family, predicts survival of patients with endometrial cancer of endometrioid subtype. Gynecol Oncol 2009;112:229-34.

48. Chien W, Kumagai T, Miller CW, et al. Cyr61 suppresses growth of human endometrial cancer cells. J Biol Chem 2004;279:53087-96.

49. Gery S, Virk RK, Chumakov K, et al. The clock gene Per2 links the circadian system to the estrogen receptor. Oncogene 2007:26:7916-20.

50. Maeta N, Osaki M, Shomori K, et al. CYR61 downregulation correlates with tumor progression by promoting MMP-7 expression in human gastric carcinoma. Oncology 2007;73:118-26.

51. Sin WC, Bechberger JF, Rushlow WJ, et al. Dose-dependent differential upregulation of CCN1/Cyr61 and CCN3/NOV by the gap junction protein Connexin43 in glioma cells. J Cell Biochem 2008;103:1772-82.
52. Bleau AM, Planque N, Perbal B. CCN proteins and cancer: two to tango. Front Biosci 2005;10:998-1009.

53. Manara MC, Perbal B, Benini S, et al. The expression of ccn3(nov) gene in musculoskeletal tumors. Am J Pathol 2002;160:849-59.

54. Heizmann CW. S100B protein in clinical diagnostics: assay specificity. Clin Chem 2004;50:249-51.

55. Harpio R, Einarsson R. S100 proteins as cancer biomarkers with focus on S100B in malignant melanoma. Clin Biochem 2004;37:512-8.

56. Davies MP, Rudland PS, Robertson L, et al. Expression of the calcium-binding protein S100A4 (p9Ka) in MMTV-neu transgenic mice induces metastasis of mammary tumors. Oncogene 1996;13:1631-7.

57. Lloyd BH, Platt-Higgins A, Rudland PS, et al. Human S100A4 (p9Ka) induces the metastatic phenotype upon benign tumor cells. Oncogene 1998;17:465-73.

58. Suzuki F, Oridate N, Homma A, et al. S100A2 expression as a predictive marker for late cervical metastasis in stage I and II invasive squamous cell carcinoma of the oral cavity. Oncol Rep 2005;14:1493-8.

59. Kyriazanos ID, Tachibana M, Dhar DK, et al. Expression and prognostic significance of S100A2 protein in squamous cell carcinoma of the esophagus. Oncol Rep 2002;9:503-10.

60. Tsai ST, Jin YT, Tsai WC, et al. S100A2, a potential marker for early recurrence in early-stage oral cancer. Oral Oncol 2005;41:349-57.

61. Moog-Lutz C, Bouillet $\mathrm{P}$, Regnier $\mathrm{CH}$, et al. Comparative expression of the psoriasin (S100A7) and S100C genes in breast carcinoma and co-localization to human chromosome 1q21-q22. Int J Cancer 1995;63:297-303.

62. Salama I, Malone PS, Mihaimeed F, et al. A review of the $\mathrm{S} 100$ proteins in cancer. Eur J Surg Oncol 2008;34:357-64.

63. Rafii S, Lyden D. S100 chemokines mediate bookmarking of premetastatic niches. Nat Cell Biol 2006:8:1321-3.

64. Kondo A, Sakaguchi M, Makino E, et al. Localization of S100C immunoreactivity in various human tissues. Acta Med Okayama 2002;56:31-4.

65. Memon AA, Sorensen BS, Meldgaard P, et al. Down-regulation of S100C is associated with bladder cancer progression and poor survival. Clin Cancer Res 2005;11:606-11.

66. Rehman I, Azzouzi AR, Cross SS, et al. Dysregulated expression of S100A11 (calgizzarin) in prostate cancer and precursor lesions. Hum Pathol 2004;35:1385-91.

67. Cross SS, Hamdy FC, Deloulme JC, et al. Expression of S100 proteins in normal human tissues and common cancers using tissue microarrays: S100A6, S100A8, S100A9 and S100A11 are all overexpressed in common cancers. Histopathology 2005;46:256-69.

68. Ohba H, Bakalova R, Muraki M. Cytoagglutination and cytotoxicity of Wheat Germ Agglutinin isolectins against normal lymphocytes and cultured leukemic cell lines--relationship between structure and biological activity. Biochim Biophys Acta 2003;1619:144-50.

69. Gabius HJ, Darro F, Remmelink M, et al. Evidence for stimulation of tumor proliferation in cell lines and histotypic cultures by clinically relevant low doses of the galactoside-binding mistletoe lectin, a component of proprietary extracts. Cancer Invest 2001;19:114-26.

70. Mizuhashi S, Nakamura K, Mori Y, et al. Insulin allergy and immunologic insulin resistance caused by interleukin- 6 in a patient with lung cancer. Diabetes Care 2006;29:1711-2

71. Seow A, Ng DP, Choo S, et al. Joint effect of asthma/atopy and an IL-6 gene polymorphism on lung cancer risk among lifetime non-smoking Chinese women. Carcinogenesis 2006;27:1240-4.

72. Gao SP, Mark KG, Leslie $\mathrm{K}$, et al. Mutations in the EGFR kinase domain mediate STAT3 activation via IL-6 production in human lung adenocarcinomas. J Clin Invest 2007;117:3846-56.

73. Frolova O, Benito J, Brooks C, et al. SL-401 and SL-501, targeted therapeutics directed at the interleukin- 3 receptor, inhibit the growth of leukaemic cells and stem cells in advanced phase chronic myeloid leukaemia. Br J Haematol 2014.

74. Saleem M, Kweon MH, Johnson JJ, et al. S100A4 accelerates tumorigenesis and invasion of human prostate cancer through the transcriptional regulation of matrix metalloproteinase 9. Proc Natl Acad Sci U S A 2006;103:14825-30.

75. Radestock Y, Willing C, Kehlen A, et al. Relaxin enhances S100A4 and promotes growth of human thyroid carcinoma cell xenografts. Mol Cancer Res 2010;8:494-506.

76. Qu W, Xiao J, Zhang H, et al. B19, a novel monocarbonyl analogue of curcumin, induces human ovarian cancer cell apoptosis via activation of endoplasmic reticulum stress and the autophagy signaling pathway. Int J Biol Sci 2013;9:766-77.

77. Jensen MM, Halekoh U, Stokes CR, Lauridsen C. Effect of maternal intake of organically or conventionally produced feed on oral tolerance development in offspring rats. J Agric Food Chem. 2013 May 22;61(20):4831-8. doi: 10.1021/jf305454c. Epub 2013 May 10.

78. Protein is an important but undervalued macronutrient in the nutritional care of patients with cystic fibrosis. Engelen MP, Com G, Deutz NE. Curr Opin Clin $\begin{array}{lllll}\text { Nutr } & \text { Metab } \quad \text { Care. } 2014 \quad \text { Nov; } 17(6): 515-20 . & \text { doi: }\end{array}$ 10.1097/MCO.0000000000000100.

79. Diet and lifestyle factors interact with MAPK genes to influence survival: the Breast Cancer Health Disparities Study. Slattery ML, Hines LH, Lundgreen A, Baumgartner KB, Wolff RK, Stern MC, John EM. Cancer Causes Control. 2014 Sep;25(9):1211-25. doi: 10.1007/s10552-014-0426-y. Epub 2014 Jul 4. 